\title{
Some Observations on François Recanati's Mental Files
}

\author{
Annalisa Coliva \\ COGITO, University of Modena \& Reggio Emilia
}

Delia Belleri

COGITO, University of Bologna

François Recanati's Mental Files provides a new and thought-provoking account of the nature and structure of singular thought. According to Recanati, mental files are non-descriptive modes of presentation and are used to tackle a variety of philosophical issues. In this contribution, we will provide a brief overview of Recanati's work and a critical assessment of some of its main theses.

\section{Background and overview}

Descriptivism is 'the view that our mental relation to individual objects goes through properties of those objects' $(3)^{1}$, that is, we think about singular objects only insofar as we ascribe properties to them. The view draws from Frege the distinction between reference and sense or mode of presentation. The components of our thoughts are senses, which are modes of presentation conceived of as descriptive, i.e. as characterising an object as the only bearer of a certain property: for example 'the morning star' or 'the evening star'.

In contrast, Singularism says that 'our thought is about individual objects as much as it is about properties. Objects are given to us directly, in experience' (4). One of the main problems of Singularism comes with cases of misidentification: suppose Charles believes that Mont Blanc is 4,000 metres high; one day, he sees a mountain

${ }^{1}$ If not otherwise specified, all references are to Recanati 2012. 
and forms the belief that it is less than 4,000 meters. Unbeknownst to him, that mountain is Mont Blanc. Since Mont Blanc occurs directly in both of Charles' thoughts, he counts as irrational for he has contradictory beliefs about the same object. Singularism has to invoke something like modes of presentation in order to be able to say that Charles' thoughts are not inconsistent, for Mont Blanc is really 'presented differently' in each thought. But is it possible to combine Singularism with modes of presentation without falling prey to Descriptivism?

Recanati's book aims at providing a positive answer to this question, thus arguing for the following combination of elements: (a) a Singularist conception of thought about individual objects; (b) the sense/reference distinction; (c) a non-Descriptivist notion of sense or mode of presentation. To achieve this result, he introduces the notion of mental files: these are modes of presentation for individual objects which, however, are not based on descriptions but rather on acquaintance relations. A relation is of acquaintance just in case it is epistemically rewarding, i.e. it enables one to acquire information from an object. Perception is a paradigm here, for it allows information to flow directly from the object to the mind. However, other kinds of 'mediated' acquaintance, through communication or contextual relations, are also possible (35-36).

Mental files thus conceived are, for one thing, repositories of information - which may or may not be veridical - about a certain object. For another, they are analogous to singular terms in that they refer to the object itself. So, for instance, Charles' being perceptually acquainted with Mont Blanc triggers the creation of a mental file, which (a) can store a certain amount of information - or misinformation (e.g. 'the mountain I see', 'the world's tallest mountain') in the form of a list of predicates and (b) refers to Mont Blanc itself. Note that, according to Recanati, the file's reference is not determined by the information contained in it. What mental files refer to

'is not determined by properties which the subject takes the referent to have (i.e. by information — or misinformation — in the file), but through the relations on which the files are based. The reference is the entity we are acquainted with (in the appropriate way), not the entity which best 'fits' information in the file.' (35) 
The mechanism of reference of mental files is modelled upon Kaplan's conception of indexicality in formal semantics. Mental file types are said to have a 'character', i.e. a rule setting the conditions at which the file itself should be tokened in one's mind, which requires different epistemically rewarding relations to be instantiated (59-60). In the case of 'I', for instance, the file should be tokened only when the relation of (the referent's) being identical with the producer of the token obtains, or is presumed to obtain (61). Note that the subject need not think about the obtaining of such relation while she creates a token of the file in her mind $(248$, note 4,251$)$. If the file refers, then it has a 'content', i.e. it contributes an individual to the truthconditions of the thought - as in the case of 'I'. If it does not, the file nevertheless counts as a genuine component of the thought - with 'intentional' features only (63-64, 246-7).

\section{Critical assessment}

Mental files, as Recanati conceives them, appear as multifarious and versatile objects. They can be used to approach in an original and challenging way many philosophical puzzles, ranging from informative identities to the communication of perspectival contents. Yet their nature and workings remain relatively unclear at least at places.

For instance, there seem to be several ambiguities in the way mental files are presented. On the one hand, we are told that they are singular Fregean senses, that determine the referents they stand for. On the other, we are told that they are similar to Fodor's terms in the language of thought. However, Fodor's concepts are only syntactically different and do not contain any semantically relevant material apart from their referent, nor is the latter determined by sense. Furthermore, we are told that mental files are singular senses but then they are used to store any kind of information. This would be fine as long as the latter didn't serve any semantic purpose, but, as the discussion of the mental file SELF in connection with the problem of its communication will presently make apparent, it is unclear whether this is so.

Another aspect of Recanati's proposal that is not entirely clear is its precise scope. For we are told that mental files are mental indexicals which depend, for their existence, on there being an epistemi- 
cally rewarding relation, in the form of acquaintance, between a subject and the object the file is a file of. On the face of it, however, this would entail that mental files are quite limited, for we do not seem to be acquainted with a lot of entities that we are nevertheless able to think about. We are not acquainted with non-existing and fictional entities; nor are we acquainted with past or future entities, let alone with abstract ones, like numbers or logical entities. Recanati, however, stresses that in the first case — the one of non-existing or fictional entities (but notice the partly confusing treatment of SUPERMAN and CLARK KENT at 197-ff) — we essay a singular thought, but we would have none (160). Rather, we would have a descriptive and therefore general thought (161). However, he also seems to say that we can report someone else's attitudes about these entities in such a way that their possession of the corresponding mental files, or at least, pseudo-singular files, would be presupposed (177, 204-5). Pseudo-singular files, however, do not seem to be equivalent to some general or descriptive thought-content. For, granted that they do not license singular thoughts, they still are to be regarded as singular in some relevant sense. In order to capture this nuance, Recanati describes subjects who entertain such files as thinking singular vehicles and not singular contents. To entertain a singular vehicle, he says, is to token a mental file which is not created on the basis of an acquaintance relation (either one that actually obtains, or one which is expected to actually obtain) (166-169). Singular vehicles however, are merely taken to provide singular reference by those who entertain them (if, e.g., they are mistaken about the existence of their referent - think of a child who believes in Santa Claus); at best, they are treated as providing singular reference (we may imagine a cautious scientist, who is not sure about the existence of the entity she is naming). In each case, theirs is only an appearance of singularity and it is not clear how one could go from an appearance of singular thought to singular thought proper, in any interesting semantic sense. So if entertaining a singular vehicle comes down to entertaining a seemingly singular thought (which is really not a singular thought, in any interesting semantic sense), we do not see how this notion could be of help. In the second case, i.e. the one of past or future entities, things are complex. As to past entities, we may be in relation to them through language, because someone was acquainted with them and a 
communicative chain was set up so as to preserve reference to these entities. In the case of future or still unknown entities, in contrast, we are tentatively told that we can already have singular thoughts about them, as long as their referents will come into existence (e.g. Newman 1) or will be discovered (e.g. Neptune for early astronomers), even though we will not be acquainted with them (164-5, $169,171)$. But this seems weird. For the causal chains, or, at any rate, the epistemically rewarding relations based on acquaintance, which will eventually be set up should have backward effects. To spell this out: in order for one to have a singular thought about an entity one will be acquainted with in the future (say, Newman 1) one must be linked with that entity in some relevant way. Following Recanati, such a link is to be regarded as an epistemically rewarding relation with a physical object (acquaintance) (20); yet if the entity in question exists only in the future, the link between the entity and the speaker must work backwards so as to ensure that singular thought is attained in the present. The problem here is that it does not seem that an epistemically rewarding relation such as acquaintance can afford this kind of connection. We may have the impression that it does, because we are able to place ourselves in an a-temporal perspective from which we can neutrally assess this relation, as it were, from a purely conceptual point of view. Yet this does not entail that, when one speaks about e.g. Newman 1 in the present, one is really related, in any epistemically rewarding way, with a physically existing individual. Finally, nothing is said about the case of abstract entities and this is partly unsatisfactory because it leaves in the dark an area of our thought that is extremely important as it accounts for some of our fundamental cognitive abilities, some of which would seem to produce singular thinking about their objects, e.g. ' 3 ', 'the positive square root of 16' (taken as referring de re to number 4), etc.

One further feature of the theory which is not entirely clear is the extent to which one's singular thoughts are transparent. Recanati disagrees with both Boghossian's and Burge's different takes on the issue of the compatibility between externalism and self-knowledge. He claims that in the following kind of inference, taking place after a slow switch between worldly mental files and their counterparts on twin Earth, 
(1) Jo once loved playing in the water.

(2) Jo does not like playing in the water now.

(3) Jo has changed.

it is not the case, contra Boghossian, that 'water' in (1) and (2) respectively refers to water and twater; nor is it the case, contra Burge, that the reference of 'water' in (2) is water, like in (1), because the reasoning initiated in (1) requires the reference of 'water' to remain stable. Rather, the reference of 'water' is confused in both cases, so it is neither water nor twater and therefore (1) and (2) are neither true nor false. Yet, according to Recanati, his account preserves transparency. For transparency has it that if there is a singular thought, then the subject would know what his thought is about. But since the premises in the inference do not satisfy the antecedent of the conditional, they cannot be taken to be a counterexample to it. Now, the intuition that no specific thought about water (or twater) is being thought is not very solid and, at any rate, it is not clear what evidence there is for thinking so. With respect to (2), where supposedly the subject is aware of Jo's behavior in the presence of some stuff resembling water, it really seems that he would be thinking a singular thought about that stuff. As it happens, it is twater, so the subject would really seem to be thinking about twater. In the case of (1) things might be a little bit more complicated, for memory is involved. But Recanati himself thinks that memory is a way of storing information about previously encountered objects, even though it also affects a transformation of the original files, since they are no longer based on perception of their referents. At any rate, it would seem that on Recanati's preferred account of the role of memory, 'water' in (1) should refer to water. If so, then transparency would not be preserved, after all. However, even if one grants Recanati the idea of confused reference in (1) and (2), this would actually entail that while the subject may be thinking of thinking a ( $t$ )water-thought in each of the premises, he would not. So, it remains unclear how the proposed solution would actually allow to compatibilize externalism and the transparency of senses, for the content of one's thoughts would still be unknown to the subject.

One further aspect of the framework presented by Recanati that deserves consideration, in our opinion, is his account of the SELF 
file and its communication. In this and other work of his, Recanati insists on the relevance, to the possession of the SELF file, of a number of epistemically rewarding relations, such as somatic proprioception, self-locating perception, memory and immediate knowledge of one's own mental states. These relations are epistemically rewarding insofar as the subject is identical to the person he receives somatic proprioceptive and self-locating perceptual information from, or to the person whose memories he is storing and whose mental states he is immediately aware of. Recanati seems to go as far as saying that these sources of information are intrinsically self-specifying (cf. 88, note 10). But, as a matter of fact, although, as a norm, one is identical to the person one is receiving the relevant kind of information from, or whose past is responsible for the memory impressions one is having, it need not always be so, when at least somatic proprioception, self-locating perception and memory are at stake. One might then deny that, when things go wrong, there is real proprioception, self-locating perception and memory. But this is not a very promising strategy as it would rule out possible counterexamples simply by definition. So, one more promising way to go would be to say that, despite the fact that these very sources of information are at work and despite the fact that they feed a subject with information which seems, at least prima facie, about himself, responsibly to exploit that information as in fact being about oneself may, at least on occasion, depend on entertaining the relevant identification components (or being prepared to do so), which might be wrong. So the identity between oneself and the person whose body is responsible for the proprioceptive/self-locating information one is receiving, or between oneself and the person whose memories one is storing, is only contingent. But if our SELF file should guarantee knowledge of its referent in all possible circumstances, for otherwise it would no longer be a SELF file, ${ }^{2}$ it cannot be based on those epistemically rewarding relations. For, in some circumstances, they would not deliver information about oneself and, if one were to entertain the relevant identification components, one would have to have a SELF file already, which allows the subject knowingly to refer to himself. Hence, we need a relation which secures the knowledgeable identity

${ }^{2}$ Cf. Anscombe 1975, Coliva 2003. 
of the subject to himself in all possible circumstances. That relation, we take it, would rather be the one between the subject and the thinker of a given occurrent thought. What this shows is that not all singular modes of presentation of a given entity, in this case the subject himself, are on a par with respect to a given file. Some would seem to be constitutive of it, like 'the thinker of this occurrent thought' for SELF. Indeed it appears as though the identity ' $\mathrm{I}=$ the thinker of this occurrent thought' - conceived as a type, not as a token — holds as a conceptual necessity and that we have a priori knowledge of it. So arguably, this kind of information will bear an especially close relation to the SELF file, such that if one were to remove it, one would lose an extremely fundamental way of identifying oneself as a subject. By contrast, the information stored in the file, which may depend on other epistemically rewarding relations or be descriptive, does not appear as constitutive of the file at issue, for the reasons just explained. These considerations seem to be worthy of thought, and perhaps Recanati's own account could benefit from them.

Moving to files and linguistic communication, recall that Recanati stresses in more than one passage that the information stored in a file should not be expected to play a semantically significant role. For instance, it should not be expected to fix the reference of the file itself — this role being fulfilled by the epistemically rewarding relation (35). But, when it comes to the problem of communicating I-thoughts, it is not entirely clear that Recanati remains faithful to this pronouncement. For he puts forward the view that in communication speaker and hearer understand each other because they share the public sense of 'I', i.e. roughly, 'the person who utters this token of 'I', which is part of their respective files SELF and HE, and correspondingly leads each subject to the SELF and HE file. In this case, some descriptive information contained in the file ('the person who utters this token of ' $\mathrm{I}$ ") is allowed to play a semantically significant role, by being what gets conveyed in communication and by being what allows subjects to latch onto the referent, via the relevant mental files. Although we appreciate the fact that, in Recanati's reconstruction of the underlying cognitive mechanism, the information does not strictly speaking fix the reference of a file - because it merely allows the subject to go to his SELF file and the hearer to go to his HE file (set up through a more direct acquaintance relation, 
such as perceptual discrimination, etc.), it is not clear to us that this mechanism completely avoids this worry. After all, the descriptive information "the person who utters this token of 'I" would be what gets conveyed in communication and what would lead each party to latch onto the appropriate file. Be that as it may, it is far from clear that this complex cognitive process is what is going on when we communicate through the use of 'I'.

Finally, in the last chapter of the book Recanati argues against a recently developed framework for the semantics of de se (and de re) thought, known as multi-centred worlds framework. ${ }^{3}$ According to this framework, the content of a de re belief like 'That man is holding a gun' - concerning, say, a threatening figure one sees in front of oneself - is a (multi) centred proposition, whose evaluation is to be effected relative to a 'base world', which comprises a possible world $w$, a time $t$, and a sequence of individuals $<_{s_{1}}, s_{2}, \ldots s_{n}>$; in the example at issue, the proposition is to be evaluated at $<_{W}, t>$ relative to individuals $<_{s_{1}}, s_{2}>$ (viz. the subject of the thought and the person the subject sees before himself). Recanati's main problem with the idea of construing centred worlds in terms of sequences of individuals is the following: one may believe to be acquainted with an individual $r$ and form a belief about $r$, where no such individual exists in the base world. In this case, there is nothing in the base world that can act as the referent of the acquaintance-based (albeit illusory) thought (258). The semantics would therefore fail to account for what is intuitively a fully-fledged de re, singular thought. In order to obviate this inconvenient, Recanati suggests the following solution: de re thoughts are to be cashed out as centred propositions, to be evaluated at a base world construed as a triple $<_{W}, i, t>$ consisting of a world, an individual and a time, which also includes a sequence of mental files, $f=$ $\langle f 1 \ldots f n>(258-9)$. Sequences of individuals are thus expunged from centred worlds, and only mental files are kept $(256,258)$. If this is so, then the files seem to acquire a strange status. On the one hand, they are mental, 'internal' objects, which act as vehicles of thought or 'mental singular terms' (viii, 35, 182, 244-5); on the other hand, they are the 'anchors' of our de re thoughts (253). These two features, however, seem difficult to reconcile: for one would think that

${ }^{3}$ See Ninan 2010 and Torre 2010. 
the objects of our de re thoughts are external to the mind, and that they do not coincide with the vehicles we use to refer to things in the world. Recanati could reply that the proper objects of our de re thoughts are really the referents of the mental files at issue - not the files themselves. This, however, just suggests that mental files alone are not enough in order to capture de re thought: individuals matter as well, and they should find their place in a suitable semantics for this kind of phenomenon. Moreover, this solution appears to us quite drastic, compared with the rather marginal problem it aims to deal with. For dismissing individuals, while retaining mental files only, causes a change in structure for all de re thoughts, even those which do have a referent. The following strategy could be adopted by the centred-worlds theorist as a way of dealing with the difficulty waived by Recanati: in the case of de re thoughts which concern no acquainted individual (due perhaps to misperception or hallucination), one could grant that the subject has no de re thought, as there is no referent, even though it seems to him to be directly and non-descriptively presented with the object. Of course this would pose a limitation to the transparency of thought, but, as we saw before, it is not clear that Recanati's own account would manage to preserve it.

\section{Conclusion}

Despite these marginal points of possible disagreement we would like to close by registering our unconditional appreciation of Recanati's attempt to reconcile Singularism with a non-descriptivist notion of mode of presentation, therefore tracing a distinction, with respect to mental files, between their reference, their relationally determined, indexical-like functional role and the information they store. We confide that this original position will play a decisive role in future debates on singular thought for many years to come. ${ }^{4}$

\footnotetext{
${ }^{4}$ Though this contribution has been discussed and conceived together, Annalisa Coliva is the author of Section 2 (save for the last paragraph), Delia Belleri of everything else. We would also like to thank Manuel García-Carpintero for very useful feed-back on a previous version of this discussion note.
} 


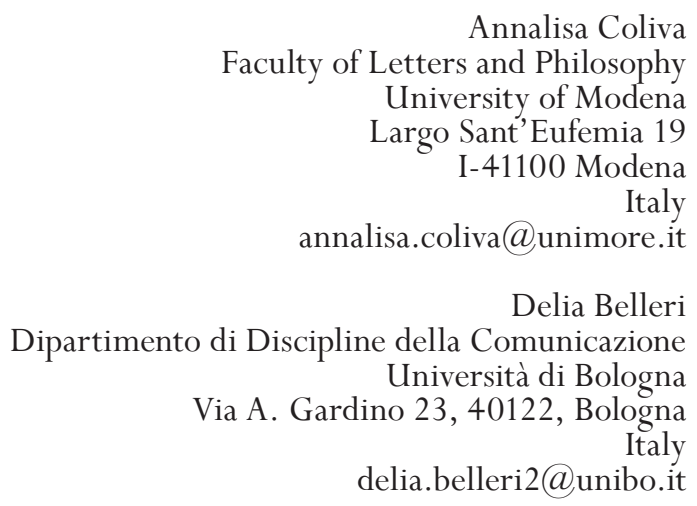

\section{References}

Anscombe, Elizabeth. 1975. The first person. In Self-Knowledge, ed. by Quassim Cassam, 140-159. Oxford: OUP.

Coliva, Annalisa. 2003. Error through misidentification, the distinction between speaker's and semantic reference and the real guarantee. The Journal of Philosophy C (8): 416-431.

Ninan, Dilip. 2010. De Se Attitudes. Ascription and Communication. Philosophy Compass 5 (7): 551-567.

Recanati, François. 2012. Mental Files. Oxford: Oxford University Press.

Torre, Stephan. 2010. Centered Assertion. Philosophical Studies 150 (1): 97-114. 